\title{
Editorial
}

\section{Trends in Classical Analysis, Geometric Function Theory, and Geometry of Conformal Invariants}

\author{
Árpád Baricz, ${ }^{1}$ Saminathan Ponnusamy, ${ }^{2}$ Matti Vuorinen, ${ }^{3}$ and Karl-Joachim Wirths ${ }^{4}$ \\ ${ }^{1}$ Department of Economics, Babes-Bolyai University, Cluj-Napoca, Romania \\ ${ }^{2}$ Department of Mathematics and Statistics, Indian Institute of Technology Madras, Chennai 600036, India \\ ${ }^{3}$ Department of Mathematics and Statistics, University of Turku, 20014 Turku, Finland \\ ${ }^{4}$ Institut for Analysis and Algebra, TU Braunschweig, 38106 Braunschweig, Germany
}

Correspondence should be addressed to Saminathan Ponnusamy; samy@iitm.ac.in

Received 11 November 2012; Accepted 11 November 2012

Copyright (C) 2013 Árpád Baricz et al. This is an open access article distributed under the Creative Commons Attribution License, which permits unrestricted use, distribution, and reproduction in any medium, provided the original work is properly cited.

The present special issue of the Journal of Abstract and Applied Analysis is devoted to trends in classical analysis, geometric function theory, and geometry of conformal mappings. In the sense of the title of this journal, we wanted to present a spectrum of research themes reaching from applied analysis to pure analysis and from applications of analysis in geometry to applications in differential equations and integral equations.

Further, our aim was to find articles on classical function theory as well as on its generalizations in several directions.

One additional aspect, that was paid attention to, is the tendency of mathematics to use computers to solve problems by new and effective algorithms. In detail, we addressed to the following themes.

That we did not forget classical pure analysis is proved by an article that considers harmonic functions on Riemann manifolds and by an article on geometry and topology of Banach spaces.

The classical geometric function theory is represented by an article on univalence criterions associated with the nth derivative.

The relationships between conformal mappings and integral equations are in the scope of two articles, where algorithms are proved to compute the mappings of unbounded multiply connected as well as bounded multiply connected regions onto slit regions.

Other old themes of classical function theory are entire functions for which we publish a paper on uniqueness theorems for monomials of entire functions.

Concerning the generalizations of classical analysis, we incorporated a paper on the stability of solutions of fractional differential equations and two papers on harmonic mappings, specially one on the general theory of log-harmonic mappings and one on certain classes of harmonic mappings defined by convolutions.

The papers on the generalizations of holomorphic functions are completed by a longer article on the distribution of zeros and poles of the rational approximants of a nonholomorphic function on an interval.

The aspect of new algorithms is addressed in an article on Hermite interpolation using Möbius transformations of planar Pythagorean-hodograph cubics and in a paper where algorithms to calculate inverse Z-transforms on the unit disc by number-theoretical methods are proved.

We hope that in this broad variety of analytic themes many researchers can find something interesting and new.

Árpád Baricz Saminathan Ponnusamy Matti Vuorinen Karl-Joachim Wirths 


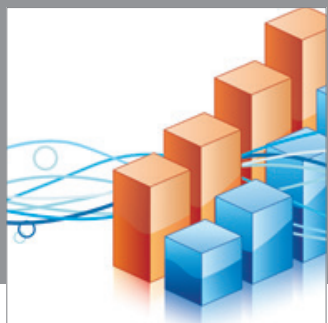

Advances in

Operations Research

mansans

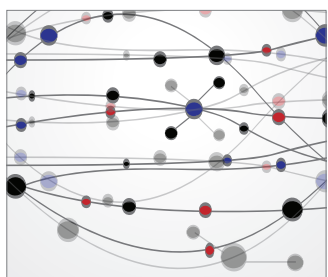

The Scientific World Journal
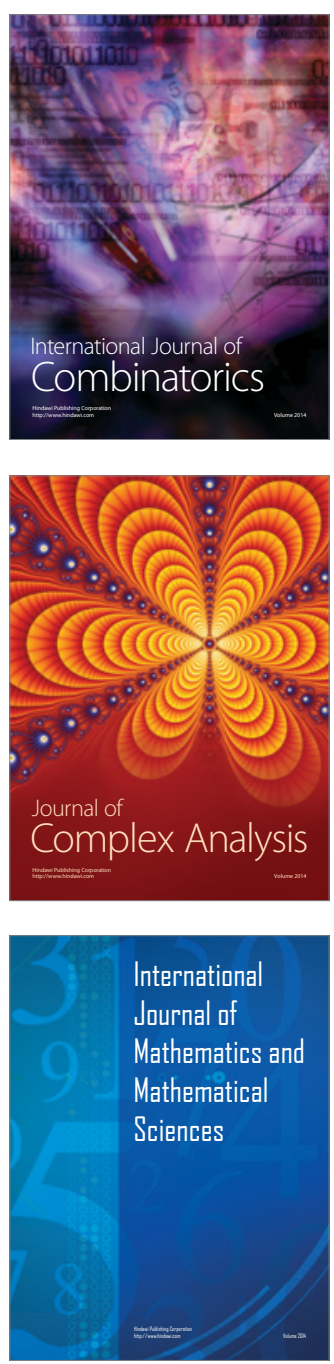
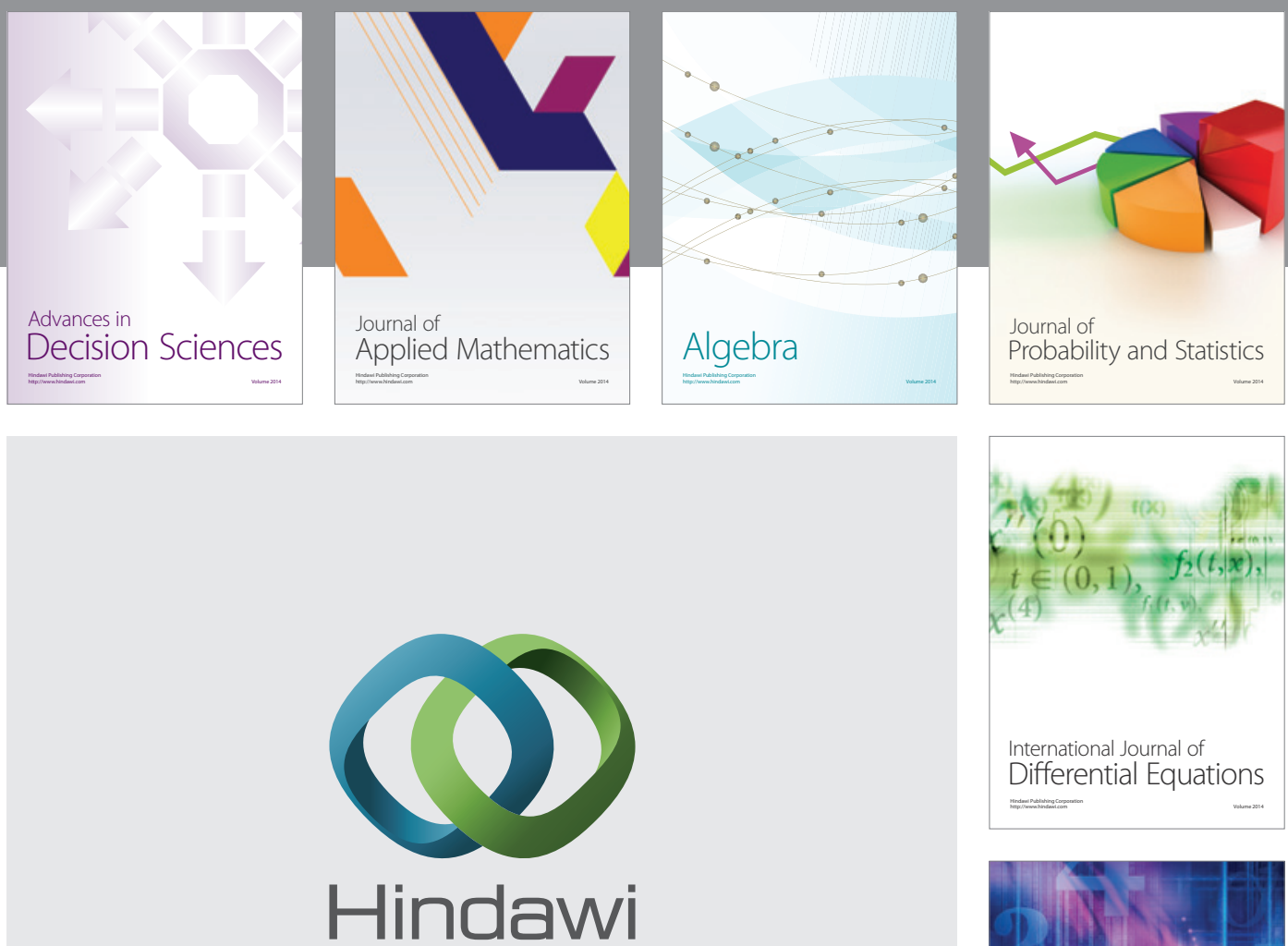

Submit your manuscripts at http://www.hindawi.com
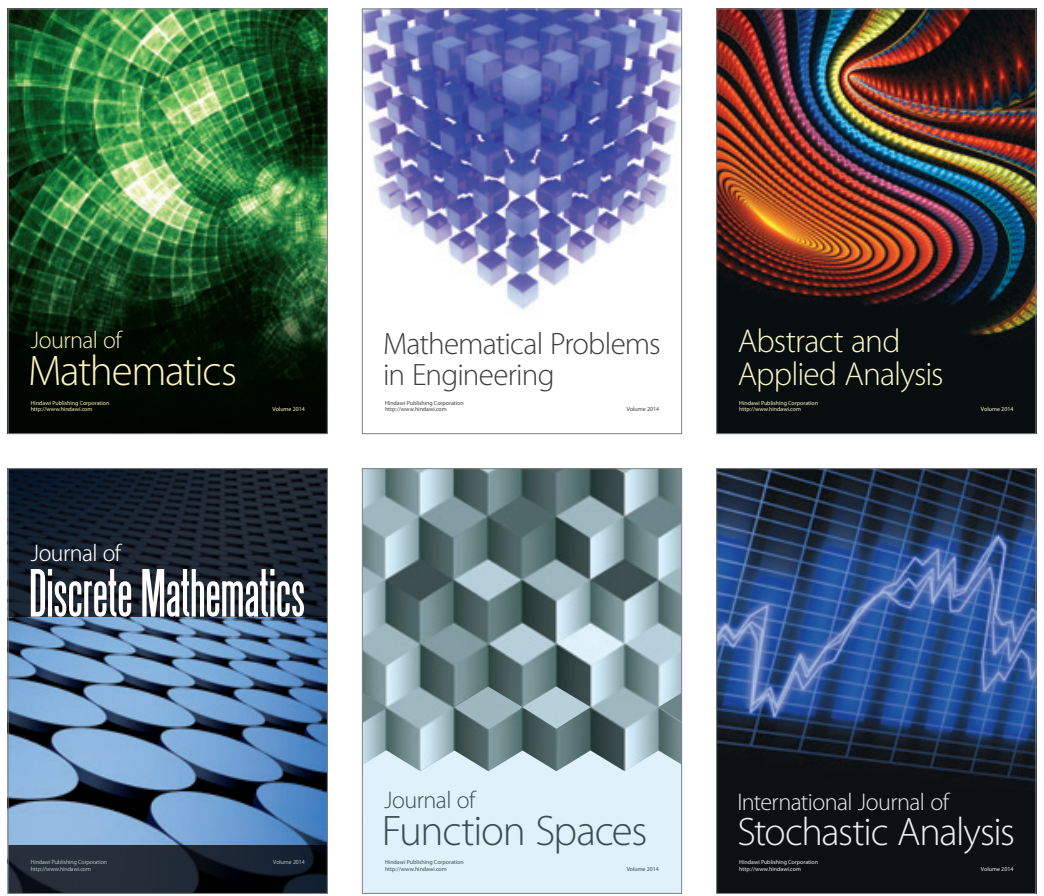

Journal of

Function Spaces

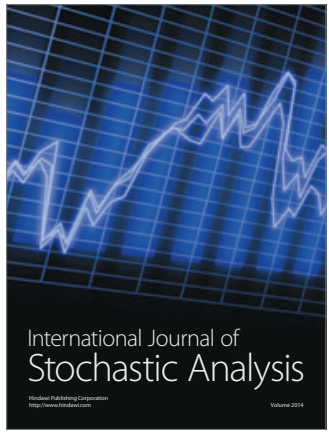

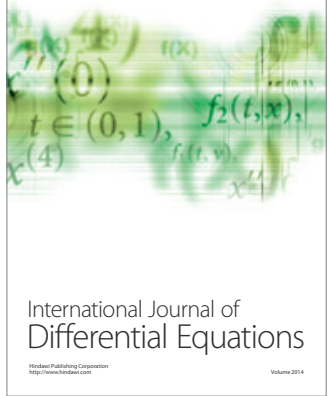
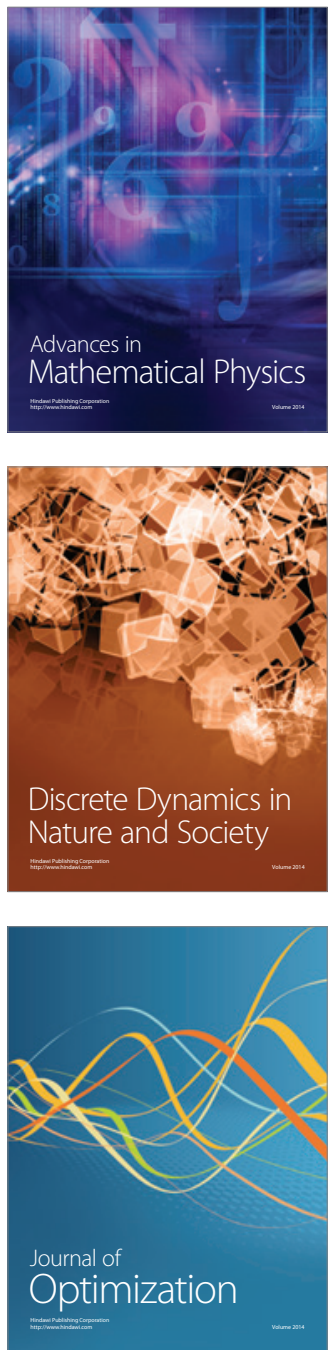